\title{
Reporte técnico de los resultados del Primer Taller Panamericano de Geomagnetismo (PANGEO)
}

\author{
J. Esteban Hernández-Quintero*
}

Recibido en julio de 2013; aceptado en septiembre de 2013

\begin{abstract}
The natural expression of magnetism in our planet has been studied for hundreds of years. Since the first attempts to describe this phenomenon, found new expressions that have kept over time within the scientific scenario.

At present, the study of geomagnetism is taking great relevance. Natural disasters such as earthquakes (L'aquila, April 2009; Haití, January 2010; Chile, April 2012; Japan, March 2012; Mexicali, April 2010); volcanic eruptions such as in México (Popocatépetl 2012), Ecuador (Tungurahua 2012) or Guatemala (Pacaya 2012); landslides, flooding or Space Weather influence in human life, are issues of great impact in our contemporary society.

The aim with this paper is to report the work done during the First Panamerican Workshop on Geomagnetism (sponsored by the American Institute of Geography and History, PAIGH). The experiences of Latin American participating countries on mitigation or prevention of natural hazards were exposed during this workshop.

The potential relationship between such issues and geomagnetism and the usefulness of this discipline to support new geophysical methodologies were the driving task.

Finally, the conclusions discussed among participating countries are reported. Among others, no less important is the continuous training of specialists in the field and finding the study of natural hazards with the help of well updated and diversified technologies with emphasis on geomagnetism.

Key words: Geomagnetism, Latin America, Geomagnetic Observatories, Geohazards.
\end{abstract}

\section{Resumen}

La expresión natural del magnetismo en nuestro planeta ha sido objeto de estudio desde hace cientos de años. Desde los primeros intentos de describir este fenómeno,

* Departamento de Geomagnetismo y Exploración Geofísica, Instituto de Geofísica, Universidad Nacional Autónoma de México (UNAM). 
se han encontrado nuevas manifestaciones que lo han mantenido a través del tiempo dentro del escenario científico.

En la actualidad, el estudio del Geomagnetismo está tomando gran relevancia. Los desastres naturales tales como terremotos (L'aquila, abril 2009; Haití, enero 2010; Chile, abril 2012; Japón, marzo 2012; Mexicali, abril 2010), erupciones volcánicas como en México (Popocatépetl 2012), Ecuador (Tungurahua 2012) o Guatemala (Pacaya 2012), deslizamientos de tierra, inundaciones o la influencia del clima espacial en la vida humana, son temas de gran impacto en nuestra sociedad contemporánea. Se busca con este trabajo reportar los trabajos desarrollados durante el Primer Taller Panamericano de Geomagnetismo (auspiciado por el Instituto Panamericano de Geografía e Historia). Exponer las experiencias de los países de América que se presentaron en este taller con el objetivo de hacer una evaluación en América Latina del quehacer en esta disciplina, con fines de mitigación o prevención de riesgos naturales con el apoyo de nuevas metodologías geofísicas. Finalmente, se menciona la capacitación continua de personal altamente especializado para el estudio de los riesgos naturales con la ayuda de la tecnología bien actualizada y diversificada con énfasis en el geomagnetismo.

Palabras clave: Geomagnetismo, Latinoamérica, observatorios geomagnéticos, riesgos geológicos.

\section{Introducción}

El Instituto Panamericano de Geografía e Historia (IPGH) ha sido una institución pionera y protagonista en el campo que nos ocupa en este trabajo. El interés por el desarrollo panamericano de la disciplina del geomagnetismo se ha reflejado desde hace más de 20 años con proyectos desarrollados principalmente por la comunidad latinoamericana para mantener y sustentar una disciplina que debe mantenerse en el continente.

La fase principal del proyecto GEOF. 052012 (PANGEO) se desarrolló entre el 28 de octubre y el 2 de noviembre de 2012. Su objetivo está basado en el espíritu de cooperación entre los países miembros del IPGH y otros países invitados que desde la década de los noventa se ha venido dando: la cooperación técnica y académica para desarrollar proyectos conjuntos entre países; así como la libre circulación de las ideas y las tecnologías necesarias para un desarrollo integral del geomagnetismo en el continente americano.

El desarrollo de este Taller se llevó a cabo en Puerto Vallarta, Jalisco (México), con la valiosa colaboración y apoyo de la Unión Geofísica Mexicana, el Taller se desarrolló dentro del marco de la Reunión Anual 2012 de esta asociación civil mexicana. Esto dio oportunidad a los participantes de la PANGEO acercarse a investigadores y especialistas en las ciencias de la Tierra del continente, ya que en su 
versión 2012; este evento concentró a más de novecientos participantes internacionales.

La dinámica de este taller se basó en tres modalidades de participación: las reuniones de trabajo plenarias entre los representantes de los siete países participantes (cerrado a PANGEO); las reuniones planificadas entre dos o más participantes con objeto de plantear la solución de proyectos específicos (cerrado a PANGEO); y la sesión plenaria de charlas (no se realizaron sesiones de carteles) abierta además a la comunidad de la Unión Geofísica Mexicana para difundir las actividades ligadas al geomagnetismo puro y sus aplicaciones más recientes.

La sesión de charlas se caracterizó por la nutrida asistencia a los trabajos presentados; temas clásicos del geomagnetismo como aquellos relacionados con el Electrochorro Ecuatorial de Sudamérica, la cartografía del campo magnético principal en distintos países, o el efecto lunar sobre el campo geomagnético, fueron tratados. Otras líneas muy actuales de investigación como el magnetismo asociado a volcanes activos; así como a la salud humana presentaron particular interés.

La participación de estudiantes en este tema fue muy importante ya que aquellos con avances importantes en temas de tesis de licenciatura, maestría o doctorado; fueron analizados, discutidos y calificados con apego estricto a la metodología académica que exige el foro.

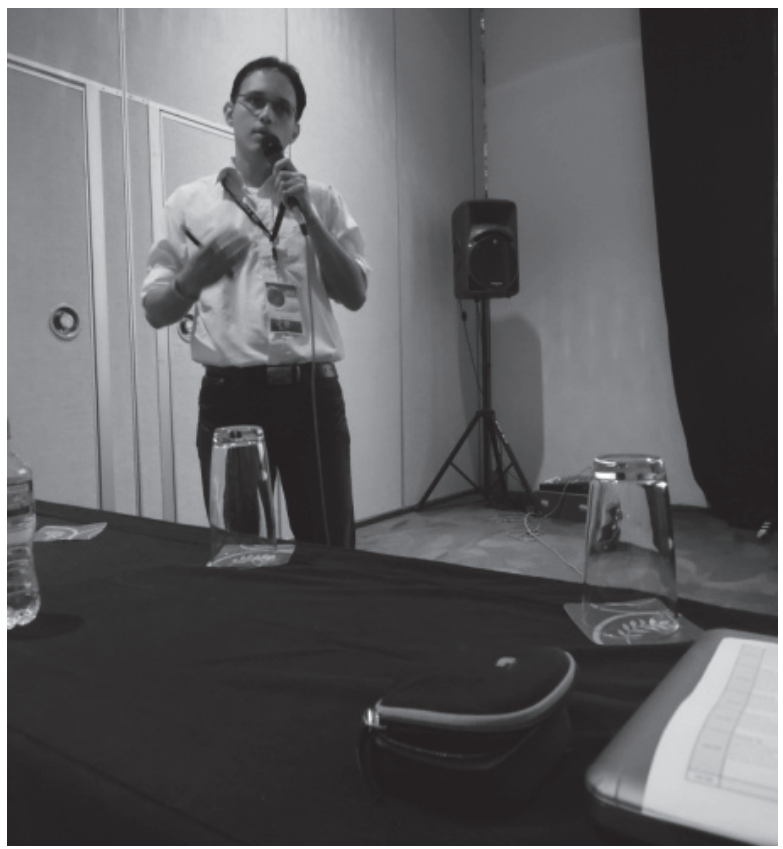

Edwin Camacho (Venezuela). 


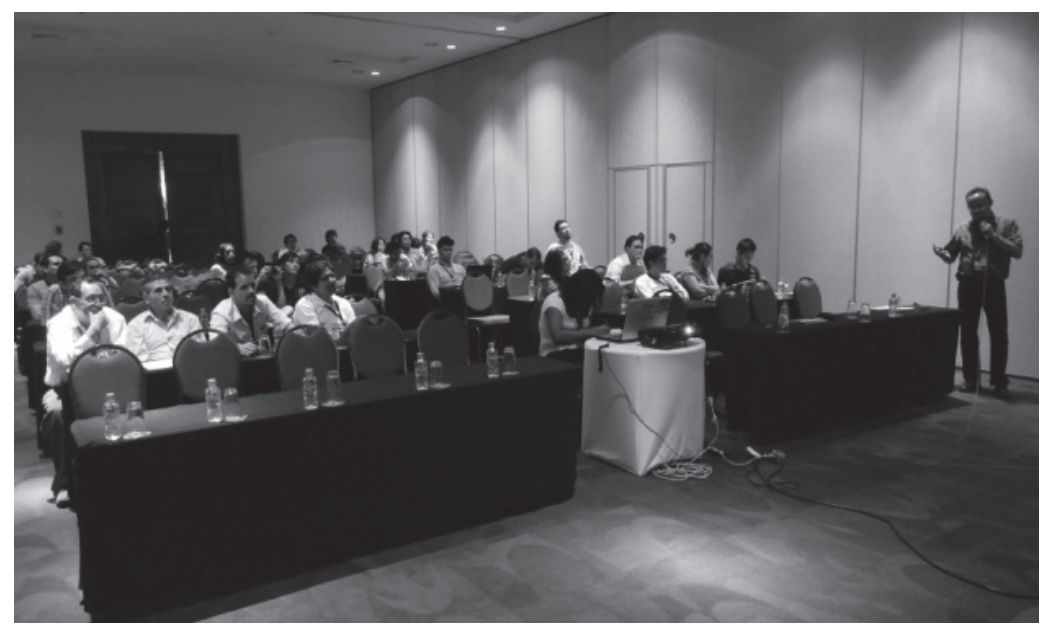

Sesión de trabajo.

En la reunión final plenaria de este taller, se reunieron representantes de los países participantes: Brasil, Perú, Venezuela, Costa Rica, Uruguay, Cuba y México. Las conclusiones de este Taller pueden resumirse en los siguientes puntos:

- Brasil se propuso como organizador del Segundo Taller Panamericano de Geomagnetismo (PANGEO II) a realizarse en Río de Janeiro.

- El uso del correo electrónico y las redes sociales como una opción para la comunicación entre los participantes, se estableció como una actividad potencial a desarrollar.

- Se propuso continuar fomentando la participación estudiantil en el área de geomagnetismo en América Latina así como el intercambio mediante la realización de talleres o capacitación en algún tema puntual en un país de esta región.

- Permitir la libre circulación de las ideas y las tecnologías necesarias para un desarrollo integral del geomagnetismo entre los países miembros; mediante la participación en foros globales relacionados con el tema, como lo es la Asamblea Científica de la Asociación Internacional de Geomagnetismo y Aeronomía (IAGA, por sus siglas en inglés), que se llevó a cabo del 21 al 31 de agosto de 2013 en Mérida, Yucatán (México).

Varios artículos presentados en este número, corresponden con uno de los objetivos del Taller. Algunos de los proyectos que se iniciaron en este Taller como un incentivo a mediano plazo, es la realización de cursos especializados para la capacitación de los profesionales que comienzan a incurrir en la disciplina como lo es el caso de los nuevos observatorios en Uruguay y Venezuela. 


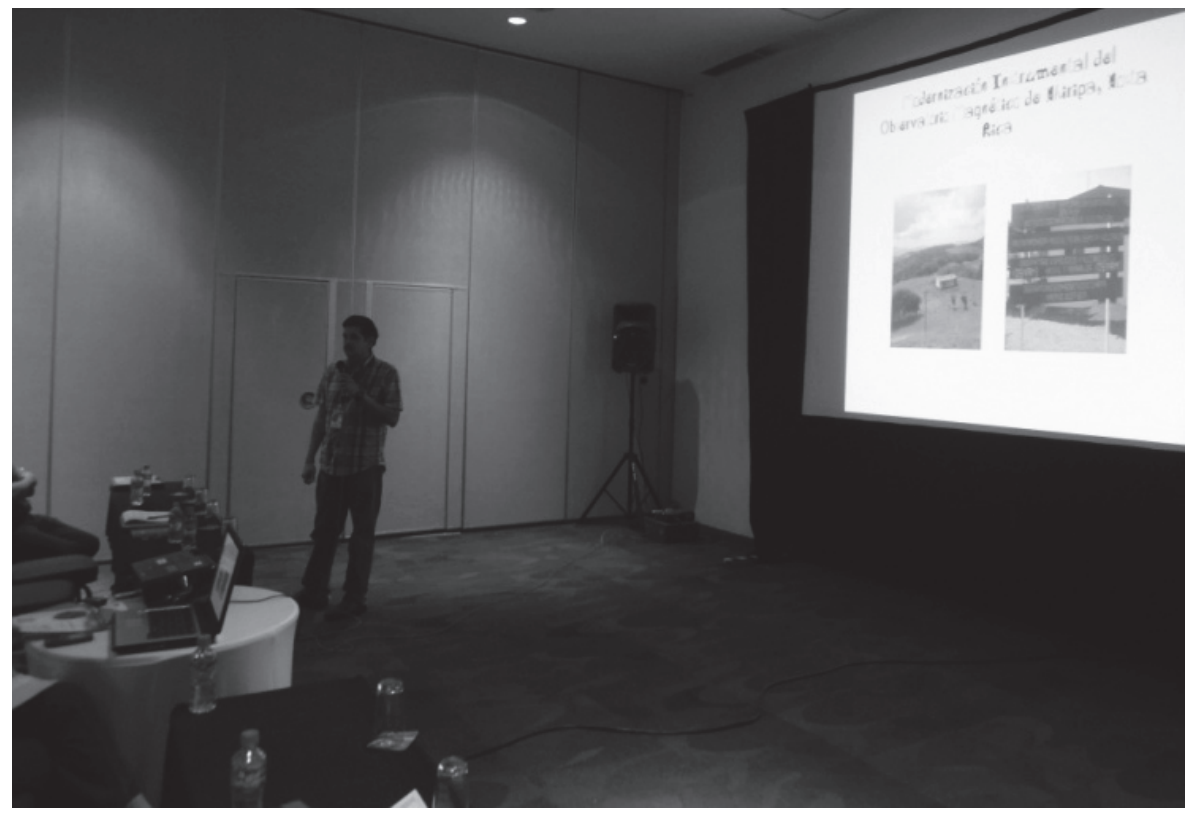

Jorge Brenes (Costa Rica).

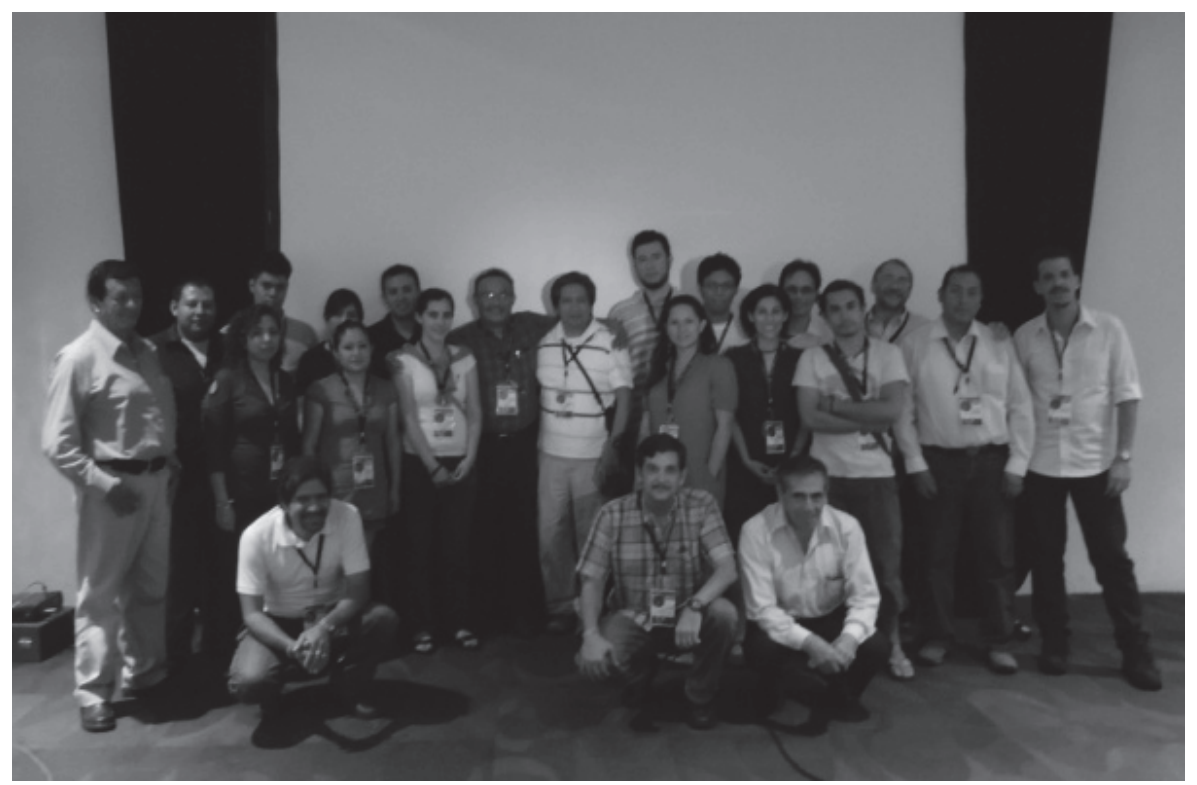

Participantes PANGEO. 
Otra área por desarrollar es la actualización de la cartografía magnética en algunos países miembros como es el caso de Costa Rica; bajo la asesoría de los observatorios en México (Teoloyucan), y Perú (Huancayo).

Finalmente, es de especial importancia agradecer en primer lugar al Instituto Panamericano de Geografía e Historia, así como a la Universidad Nacional Autónoma de México (a través del Instituto de Geofísica); por su interés, apoyo y sobre todo el compromiso que a través de los años han logrado consolidar mediante una actitud positiva de cooperación entre los países de las Américas. Así mismo, a la Unión Geofísica Mexicana por compartir los formatos organizativos que hicieron de este evento un éxito para todos los involucrados.

\section{Antecedentes de proyectos sobre geomagnetismo en América Latina}

Actualmente el mecanismo más extendido para el estudio del campo magnético terrestre en su superficie son los observatorios magnéticos distribuidos en poco más de 20 millones de kilómetros cuadrados, que conforma América Latina. Aunque son insuficientes aun para estudiar esta disciplina eficientemente, en el pasado reciente, el panorama era poco menos alentador; con tan solo trece observatorios geomagnéticos en 1990 (Hernández-Quintero et al., 2009). En 1993, se inicia una serie de acciones que llevaron a una integración muy importante en el geomagnetismo en América.

\section{Tabla 1}

Periodo de desarrollo (1990-2012) de proyectos de alcance latinoamericano que han determinado la evolución actual del Geomagnetismo en el área

\begin{tabular}{ll}
\hline \multicolumn{1}{c}{ Año } & \multicolumn{1}{c}{ Eventos auspiciados por el IPGH } \\
\hline 1990 & Primera Calibración de Observatorios en América Latina \\
1993 & Primera Escuela Latinoamericana de Geomagnetismo (Fúquene, Colombia) \\
1994 & Observaciones geomagnéticas durante eclipses solares (México-Brasil) \\
1995 & Segunda Escuela Latinoamericana de Geomagnetismo (Taxco, México) \\
1996 & Primer Taller Internacional de Instrumentos en Geomagnetismo \\
& (Teoloyucan, México) \\
2000 & Tercera Escuela Latinoamericana de Geomagnetismo (Huancayo, Perú) \\
2004 & Cuarta Escuela Latinoamericana de Geomagnetismo (Chiripa, Costa Rica) \\
2005 & Quinta Escuela Latinoamericana de Geomagnetismo (Juriquilla, México) \\
2007 & Primera Reunión Intermagnet (Ciudad Universitaria, México) \\
2012 & Sexta Escuela Latinoamericana de Geomagnetismo “Luiz Muniz Barreto" \\
& Passouras, Brasil)
\end{tabular}



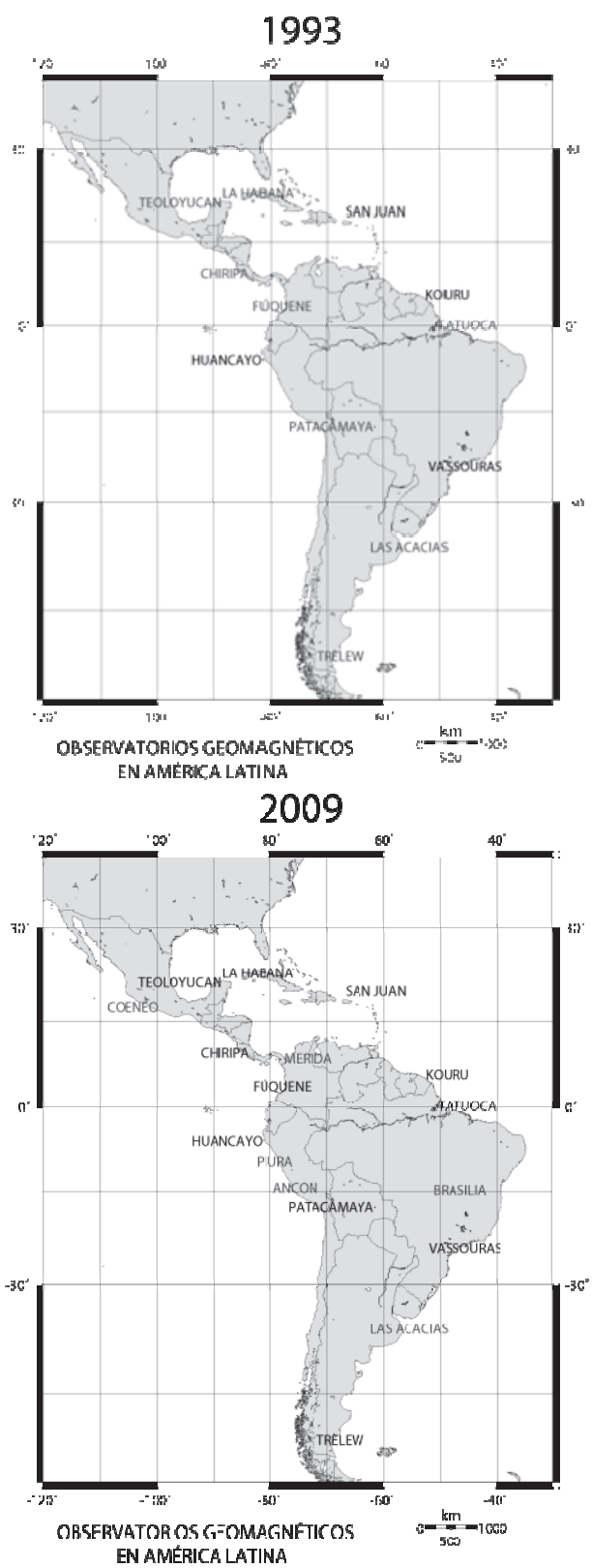

Figura 1. Mapas comparativos del desarrollo de observatorios geomagnéticos en América Latina entre 1993 y 2009. Este importante incremento de estaciones muestra el impacto de las acciones tomadas desde 1990 en proyectos en donde el IPGH ha participado. 
Gracias al esfuerzo de instituciones como el Instituto Panamericano de Geografía e Historia, el Observatorio Nacional de Río de Janeiro, y la Universidad Nacional Autónoma de México, fue a partir de 1990 cuando se logran identificar los problemas principales de cada uno de estos observatorios (Godoy, 1996) y se establece una estrategia para modernizar las estaciones existentes y proponer un programa de desarrollo. Este impulso inicial dio por resultado una serie de proyectos entre 1990 y 2012 en donde el IPGH participó de manera muy importante y determinante (Tabla 1).

\section{América Latina y la ciencia del Geomagnetismo}

El esfuerzo sostenido durante 20 años (1993-2013) para establecer las condiciones de desarrollo de la ciencia del geomagnetismo en América Latina, han reflejado ciertas fortalezas y debilidades de aquellos países que tienen las condiciones mínimas para un potencial desarrollo.

Se tienen ejemplos en Perú, Brasil y México (los observatorios en estos países están cerca del centenario de operación) en donde han captado de manera exitosa el apoyo tecnológico del mundo desarrollado en esta disciplina mediante la capacitación de profesionales. Tomando los casos como Perú-Japón o México-Estados Unidos, la transferencia de las tecnologías han sido exitosas por el interés del mundo desarrollado en estudiar la corriente de electrochorro sobre Perú, u otros fenómenos específicos.

El análisis cuidadoso de los eventos que se han llevado a cabo a nivel latinoamericano, en este camino pretende dar énfasis a una participación de carácter panamericano bajo políticas de desarrollo tales como la presentación de resultados del Geomagnetismo asociado a la actividad volcánica (Cifuentes, 2009), en sismología (Thomas et al., 2009; Cifuentes-Nava et al., 2007), e incipientemente en el cambio climático (Love, 2010); esto con el fin de abrir nuevos horizontes para el estudio de las complejas expresiones de los riesgos naturales asociados.

Es necesaria la formulación de nuevas propuestas de investigación y evaluación relacionadas con el efecto del cambio climático en nuestra disciplina. La posibilidad de exponer estudios que documenten la mitigación o prevención de riesgos naturales con el apoyo de nuevas metodologías geofísicas íntimamente ligadas con el geomagnetismo, debe de tomarse con gran interés por la comunidad.

La misión de los observatorios geomagnéticos en América debe estandarizarse en la medida de los intereses nacionales de cada país miembro. Es necesario proveer registros continuos de campo magnético lo más duraderos posible, para la difusión de sus productos a entidades gubernamentales, académicas e instituciones privadas. Esto con el fin principal de desarrollar la investigación de la naturaleza de las variaciones geomagnéticas para lograr la mitigación de riesgos. 
Establecer conciencia acerca de modelos teóricos del campo geomagnético global y la cooperación de la comunidad científica internacional en combinación con las distintas misiones satelitales que miden el campo geomagnético. La integración de esta comunidad a Intermagnet (un consorcio internacional de observatorios geomagnéticos) y a otras agencias con metas similares como la International Asociation of Geomagnetism and Aeronomy redundará en un mejor estudio y registro del campo magnético terrestre.

La comunidad internacional está especialmente interesada en la caracterización de la compleja estructura del campo magnético en el tiempo y en el espacio, recientemente se ha iniciado un nuevo ciclo de actividad solar; la tendencia en el mundo desarrollado es registrar el fenómeno con una resolución a cada segundo. Esto implica un muestreo real de al menos $20 \mathrm{~Hz}$ para lograr tal objetivo. Sin embargo una de las grandes carencias es que casi todos los países en el área no han desarrollado la tecnología para diseñar una electrónica con este nivel.

Por otro lado, existen ejemplos de desarrollo como en el caso de Brasil, donde se están probando nuevos materiales, y diseños novedosos de magnetómetros para su uso exclusivo en latitudes de aquel país. Adicionalmente otros países como Perú o Colombia, presentan interés en la relación de los fenómenos magnéticos con erupciones volcánicas.

Para lograr un avance válido en la dirección correcta, los observatorios en América Latina lograrían un avance significativo realizando este tipo de muestreo. En la actualidad los observatorios en el área registran el campo a cada minuto (Love et al., 2007).

\section{Metodología}

Siguiendo las experiencias que se han aplicado en los talleres desde el año 1993, fue posible alcanzar los objetivos planteados mediante acciones en varios niveles:

a) La organización de talleres y congresos a nivel panamericano.

b) Una mayor participación de los especialistas en distintas asociaciones de geofísica y geomagnetismo fuera del ámbito latinoamericano.

c) La promoción de proyectos conjuntos entre dos o más países del área, con énfasis en la transferencia de tecnología para la resolución de problemas comunes.

d) La incorporación de las nuevas generaciones de especialistas en el área en este tipo de proyectos, congresos e intercambios.

En la Primera PANGEO, se aplicaron este tipo de políticas. Dado que el desarrollo del taller se hizo dentro del seno de la reunión de la Unión Geofísica Mexica- 
na, que a su vez, cuenta con la participación de especialistas no solo de México sino de países desarrollados.

La participación eficiente en charlas invitadas, conferencias y presentación de posters; dio como resultado un proceso de enseñanza-aprendizaje tanto en el plano académico, como en la práctica cotidiana de los especialistas responsables de programas de trabajo en sus respectivos países.

La participación de estudiantes de distintas entidades académicas como la Benemérita Universidad Autónoma de Puebla, el Instituto Tecnológico de Morelos, el Centro de Geociencias de Juriquilla entre otras; permitió que aquellos estudiantes en proceso de formación en licenciatura, maestría y doctorado; pudiesen conocer las alternativas de desarrollo profesional dentro de esta rama.

\section{Productos}

Al momento los productos en desarrollo del Primer Taller Panamericano de Geomagnetismo han sido en dos líneas principales:

a) El presente número de la Revista Geofisica, pública un compendio de varios de los trabajos presentados durante este Taller, se agruparon los trabajos bajo el rubro de "Artículos PANGEO", que suman un total de seis.

b) Como resultado del espíritu de cooperación entre los países participantes, se encuentran en desarrollo como mínimo, dos proyectos binacionales como lo es el caso de la Carta Magnética de Costa Rica; y la puesta en operación del Observatorio Magnético de Mérida (entre México y Venezuela).

c) Con el fin de dar continuidad a los proyectos en el área de geomagnetismo, Brasil se ha interesado en la organización del Segundo Taller Panamericano de Geomagnetismo que tendrá lugar en ese país.

\section{Conclusiones}

La Figura y la Tabla 1 son un reflejo de los logros y beneficios que se han alcanzado gracias a las acciones emprendidas en el pasado. En 2012 se obtuvo una declaración conjunta de los siete países participantes haciendo énfasis en el desarrollo necesario de la disciplina del geomagnetismo.

Es necesario continuar con este tipo de talleres que en mayor o menor grado tienen sus efectos benéficos en la comunidad latinoamericana, dado que pueden iniciarse proyectos locales o binacionales para el análisis y solución de problemas de diversa índole.

En este tenor, y de acuerdo con la evolución de los grupos de trabajo, los logros se pueden resumir en los siguientes puntos: 
a) La concientización de los profesionales relacionados con el Geomagnetismo acerca de la necesidad de incorporar nuevas herramientas a esta disciplina y además, buscar su aplicación a los riesgos naturales que afectan a la sociedad contemporánea.

b) Atraer la atención de las nuevas generaciones con una mentalidad interdisciplinaria para la mitigación de riesgos y resolución de problemas contemporáneos bajo este esquema.

c) La divulgación del trabajo de aquellos grupos que han aplicado nuevas técnicas de exploración adicionales al Geomagnetismo para el planteamiento y resolución de problemas más locales como los mencionados en el planteamiento del problema.

\section{Referencias}

Arango Galván, Claudia, 2001. Análisis temporal del campo magnético y deformación en el volcán Popocatépetl, Ingeniería-Geofísica, UNAM.

Cifuentes-Nava Gerardo, 2009. Monitoreo geomagnético del volcán Popocatépetl, tesis para obtener el grado de Maestría, Posgrado en Ciencias de la Tierra, Instituto de Geofísica, UNAM, 77 pp.

Cifuentes-Nava, G.; Cabral-Cano, E., Hernández-Quintero, J.E.; Hrvoic, I.; Wilson M. y Hoyller G., 2007. "Installation of a high resolution potassium magnetometer in the coast of Oaxaca”, Inst. Geophys. Pol. Acad. Sc., 398, 2007.

Godoy de Carvalho, Rogerio, 1996. "Report on the Calibration of Latin American Geomagnetic Observatories", Revista de Geofísica, núm. 44, Instituto Panamericano de Geografía e Historia.

Hernández Ordóñez, R. y Lora de la Fuente, C., 2003. Estudio preliminar para la instalación de un observatorio magnético en Juriquilla, Querétaro, tesis para obtener el grado de Ingeniero Geofísico, Facultad de Ingeniería, UNAM, 110 pp.

Hernández-Quintero, J.E., 2009. "Evolución del Geomagnetismo en América Latina (1990-2009)", Memorias de la 11a. Reunión de Consulta de la Comisión de Geofísica del Instituto Panamericano de Geografía e Historia, Quito, Ecuador, 26-30 de octubre de 2009.

, 2002. Interpretación global de anomalías corticales satelitales sobre la República Mexicana, tesis de Maestría, Posgrado en Ciencias de la Tierra, $164 \mathrm{pp}$.

Hernández-Quintero, J.E.; Cifuentes-Nava, G.; Caccavari-Garza A. y Flores-Soto X., 2009. "Some Considerations on Secular Variation of the Magnetic Field in Mexico", Open File Report 2009-1226, Proceedings of the XIIIth IAGA Workshop on Geomagnetic Observatory Instruments, Data Acquisition, and Processing, US Geological Survey, pp. 108-115. 
Nava Flores, M., 2005. Análisis armónico en la detección de fenómenos geomagnéticos, tesis para obtener el grado de Ingeniero Geofísico, Facultad de Ingeniería, UNAM, pp. 89.

Love, J.J., Applegate, D. y Townshend, J.B., 2008. Monitoring the Earth's Dynamic Magnetic Field, USGS Fact Sheet, pp. 2007-3092.

Love, J. y Gannon, J.L., 2010. "Movie maps of low latitude magnetic storm disturbance", Space Weather, Vol. 8.

Thomas, Jeremy N.; Love, Jeffrey J. and Johnston, Malcolm, J.S., 2009. On the reported magnetic precursor of the 1989 Loma Prieta earthquake, Physics of the Earth and Planetary Interiors, vol. 173, pp. 207-215. 